PROCEEDINGS OF THE

AMERICAN MATHEMATICAL SOCIETY

Volume 125, Number 10, October 1997, Pages 2939-2942

S 0002-9939(97)03914- 2

\title{
WEAK TYPE BOUNDS FOR A CLASS OF ROUGH OPERATORS WITH POWER WEIGHTS
}

\author{
YONG DING
}

(Communicated by J. Marshall Ash)

\begin{abstract}
In this note we show that $T_{\Omega, \alpha}$ and $M_{\Omega, \alpha}$, the fractional integral and maximal operators with rough kernel respectively, are bounded operators from $L^{1}\left(|x|^{\beta(n-\alpha) / n}, \mathbb{R}^{n}\right)$ to $L^{n /(n-\alpha), \infty}\left(|x|^{\beta}, \mathbb{R}^{n}\right)$, where $0<\alpha<n$ and $-1<\beta<0$.
\end{abstract}

\section{$\S 1$. INTRODUCTION}

Suppose that $0<\alpha<n$, and $\Omega \in L^{s}\left(S^{n-1}\right)(s \geq 1)$, where $S^{n-1}$ denotes the unit sphere of $\mathbb{R}^{n}$. Moreover, $\Omega$ is homogeneous of degree zero. We define the fractional maximal operator by

$$
M_{\Omega, \alpha} f(x)=\sup _{r>0} \frac{1}{r^{n-\alpha}} \int_{|x-y|<r}|\Omega(x-y)||f(y)| d y
$$

and the fractional integral operator by

$$
T_{\Omega, \alpha} f(x)=\int_{\mathbb{R}^{n}} \frac{\Omega(x-y)}{|x-y|^{n-\alpha}} f(y) d y .
$$

In 1971, B.Muckenhoupt and R.Wheeden [2] studied the weighted norm inequalities for $T_{\Omega, \alpha}$ with power weight. On the other hand, in 1993, S.Chanillo, D.Watson and R.Wheeden [1] proved that $T_{\Omega, \alpha}$ is of weak type $(1, n /(n-\alpha))$ under the restriction of $s \geq n /(n-\alpha)$. In this note, we use the idea in [3] to extend the result about weighted weak type $(1, n /(n-\alpha))$ for $T_{\Omega, \alpha}$ and $M_{\Omega, \alpha}$ to power weights.

Let us now formulate our results as follows.

Theorem 1. Let $0<\alpha<n,-1<\beta<0, n /(n-\alpha) \leq s \leq \infty$ and $\Omega \in$ $L^{s}\left(S^{n-1}\right)$. Then $T_{\Omega, \alpha} \quad$ is a bounded operator from $L^{1}\left(|x|^{\beta(n-\alpha) / n}, \mathbb{R}^{n}\right)$ to $L^{n /(n-\alpha), \infty}\left(|x|^{\beta}, \mathbb{R}^{n}\right)$. That is , for any $\lambda>0$ and any $f \in L^{1}\left(|x|^{\beta(n-\alpha) / n}, \mathbb{R}^{n}\right)$,

$$
\int_{\left\{x:\left|T_{\Omega, \alpha} f(x)\right|>\lambda\right\}}|x|^{\beta} d x \leq C\left(\frac{1}{\lambda} \int_{\mathbb{R}^{n}}|f(x)||x|^{\beta(n-\alpha) / n} d x\right)^{n /(n-\alpha)},
$$

where $C$ is independent of $\lambda$ and $f$.

Received by the editors January 24, 1996 and, in revised form, May 3, 1996.

1991 Mathematics Subject Classification. Primary 42B20.

Key words and phrases. Fractional integral and maximal operators, power weights.

The author was supported by NSF of Jiangxi in China. 
Theorem 2. Let $0<\alpha<n,-1<\beta<0, n /(n-\alpha) \leq s \leq \infty$ and $\Omega \in$ $L^{s}\left(S^{n-1}\right)$. Then $M_{\Omega, \alpha} \quad$ is a bounded operator from $L^{1}\left(|x|^{\beta(n-\alpha) / n}, \mathbb{R}^{n}\right)$ to $L^{n /(n-\alpha), \infty}\left(|x|^{\beta}, \mathbb{R}^{n}\right)$. That is, for any $\lambda>0$ and any $f \in L^{1}\left(|x|^{\beta(n-\alpha) / n}, \mathbb{R}^{n}\right)$,

$$
\int_{\left\{x:\left|M_{\Omega, \alpha} f(x)\right|>\lambda\right\}}|x|^{\beta} d x \leq C\left(\frac{1}{\lambda} \int_{\mathbb{R}^{n}}|f(x)||x|^{\beta(n-\alpha) / n} d x\right)^{n /(n-\alpha)},
$$

where $C$ is independent of $\lambda$ and $f$.

\section{§2. PRoOF OF THE THEOREMS}

Let us begin by giving some lemmas.

Lemma 1. Let $q>1$ and $T$ be a sublinear operator satisfying for each $a>0$ the estimate

$$
\left|\left\{\frac{a}{2} \leq|x| \leq a:\left|T\left(f \chi_{\{|x|>2 a\}}\right)(x)\right|>\lambda\right\}\right| \leq C\left(\frac{1}{\lambda} \int_{|y|>2 a}|f(y)|\left(\frac{a}{|y|}\right)^{1 / q} d y\right)^{q} .
$$

Then, if $T$ is of weak type $(1, q)$, it is also of weak type $\left(L^{1}\left(|x|^{\beta / q}\right), L^{q, \infty}\left(|x|^{\beta}\right)\right)$ for $-1<\beta<0$.

Proof. Given $f$, we now define, for each $k \in \mathbb{Z}, f_{k, 0}=f \chi_{\left\{|x| \leq 2^{k+1}\right\}}$ and $f_{k, 1}=$ $f-f_{k, 0}$. Then we can write, as usual,

$$
|T f(x)| \leq \sum_{k}\left|T f_{k, 0}\right| \chi_{I_{k}}+\sum_{k}\left|T f_{k, 1}\right| \chi_{I_{k}}=T_{0} f(x)+T_{1} f(x),
$$

where $I_{k}=\left\{x \in \mathbb{R}^{n}: 2^{k-1} \leq|x|<2^{k}\right\}$ for each $k \in \mathbb{Z}$.

If we call $\omega_{\beta}(x)=|x|^{\beta}$, we have

$$
\begin{aligned}
\omega_{\beta}\{x & \left.: T_{0} f>\lambda\right\}=\int_{\left\{x: T_{0} f>\lambda\right\}} \omega_{\beta}(x) d x \\
& \leq C \sum_{k} \omega_{\beta}\left(2^{k}\right)\left|\left\{x \in I_{k}: T_{0} f_{k, 0}>\lambda\right\}\right| \\
& \leq \frac{C}{\lambda^{q}} \sum_{k} \omega_{\beta}\left(2^{k}\right)\left(\int_{|y| \leq 2^{k+1}}|f(y)| d y\right)^{q} \\
& =\frac{C}{\lambda^{q}} \sum_{k} \omega_{\beta}\left(2^{k}\right)\left(\sum_{j \leq k+1} \int_{I_{j}}|f(y)| d y\right)^{q} \\
& \leq \frac{C}{\lambda^{q}}\left\{\sum_{j}\left[\sum_{k \geq j-1}\left(\int_{I_{j}}|f(y)| d y\right)^{q} \omega_{\beta}\left(2^{k}\right)\right]^{1 / q}\right\}^{q} \\
& =\frac{C}{\lambda^{q}}\left\{\sum_{j} \int_{I_{j}}|f(y)| d y\left[\sum_{k \geq j-1} \omega_{\beta}\left(2^{k}\right)\right]^{1 / q}\right\}^{q} \\
& \leq \frac{C}{\lambda^{q}}\left\{\sum_{j} \int_{I_{j}}|f(y)| \omega_{\beta}\left(2^{j}\right)^{1 / q} d y\right\}^{q} \leq C\left\{\frac{1}{\lambda} \int_{\mathbb{R}^{n}}|f(y)| \omega_{\beta}(y)^{1 / q} d y\right\}^{q} .
\end{aligned}
$$


Here we have used that $T$ is a weak type $(1, q)$ bounded operator and $\beta<0$. In order to estimate $T_{1}$, we make use of $(2.1)$ :

$$
\begin{aligned}
\omega_{\beta}\left\{x: T_{1} f>\lambda\right\} & =\int_{\left\{x: T_{1} f>\lambda\right\}} \omega_{\beta}(x) d x \\
& \leq C \sum_{k} \omega_{\beta}\left(2^{k}\right)\left|\left\{x \in I_{k}: T_{1} f_{k, 1}>\lambda\right\}\right| \\
& =\frac{C}{\lambda^{q}} \sum_{k} \omega_{\beta}\left(2^{k}\right)\left(\int_{|y|>2^{k+1}}|f(y)|\left(\frac{2^{k}}{|y|}\right)^{1 / q} d y\right)^{q} \\
& =\frac{C}{\lambda^{q}} \sum_{k} \omega_{\beta}\left(2^{k}\right) 2^{k}\left(\sum_{j \geq k} \int_{I_{j}}|f(y)|\left(\frac{1}{|y|}\right)^{1 / q} d y\right)^{q} \\
& \leq \frac{C}{\lambda^{q}}\left\{\sum_{j}\left[\sum_{k \leq j} \omega_{\beta}\left(2^{k}\right) 2^{k}\left(\int_{I_{j}}|f(y)|\left(\frac{1}{|y|}\right)^{1 / q} d y\right)^{q}\right]^{1 / q}\right\}^{q} \\
& =\frac{C}{\lambda^{q}}\left\{\sum_{j} \int_{I_{j}}|f(y)|\left(\frac{1}{|y|}\right)^{1 / q} d y\left[\sum_{k \leq j} \omega_{\beta}\left(2^{k}\right) 2^{k}\right]^{1 / q}\right\}^{q} \\
& \leq \frac{C}{\lambda^{q}}\left\{\sum_{j} \int_{I_{j}}|f(y)| d y \frac{1}{2^{j / q}} \omega_{\beta}\left(2^{j}\right)^{1 / q} 2^{j / q}\right\}^{q} \\
& \leq C\left\{\frac{1}{\lambda} \int_{\mathbb{R}^{n}}|f(y)| \omega_{\beta}(y)^{1 / q} d y\right\}^{q},
\end{aligned}
$$

where we have used that $\beta>-1$. This finishes the proof of Lemma 1 .

Lemma 2. Let $0<\alpha<n, \Omega \in L^{s}\left(S^{n-1}\right)$ and $s \geq 1$. Then there is a $C>0$ depending only on $n$ and $\alpha$, such that

$$
M_{\Omega, \alpha} f(x) \leq C T_{|\Omega|, \alpha}(|f|)(x) .
$$

Proof. Fix $r>0$; then we have

$$
\begin{aligned}
T_{|\Omega|, \alpha}(|f|)(x) & \geq \int_{|x-y|<r} \frac{|\Omega(x-y)|}{|x-y|^{n-\alpha}}|f(y)| d y \\
& \geq \frac{1}{r^{n-\alpha}} \int_{|x-y|<r}|\Omega(x-y)||f(y)| d y .
\end{aligned}
$$

Taking the supremum for $r>0$ on two sides of (2.3), we get

$$
T_{|\Omega|, \alpha}(|f|)(x) \geq \sup _{r>0} \frac{1}{r^{n-\alpha}} \int_{|x-y|<r}|\Omega(x-y)||f(y)| d y .
$$

This is $(2.2)$.

Now, let us turn to the proof of the theorems. In the first we consider Theorem 1. Since $T_{\Omega, \alpha}$ is weak type $(1, n /(n-\alpha))$, by Lemma 1 we only need to show that 
$T_{\Omega, \alpha}$ satisfies $(2.1)$ for $q=n /(n-\alpha)$ and any $a>0$. In fact,

$$
\begin{aligned}
& \left|\left\{\frac{a}{2} \leq|x| \leq a:\left|T_{\Omega, \alpha}\left(f \chi_{\{|x|>2 a\}}\right)(x)\right|>\lambda\right\}\right| \\
& \leq \frac{1}{\lambda^{q}} \int_{|x| \leq a}\left|T_{\Omega, \alpha}\left(f \chi_{\{|x|>2 a\}}\right)(x)\right|^{q} d x \\
& =\frac{1}{\lambda^{q}} \int_{|x| \leq a}\left|\int_{|y|>2 a} \frac{\Omega(x-y)}{|x-y|^{n-\alpha}} f(y) d y\right|^{q} d x \\
& \leq \frac{1}{\lambda^{q}}\left\{\int_{|y|>2 a}|f(y)|\left(\int_{|x| \leq a} \frac{|\Omega(x-y)|^{q}}{|x-y|^{(n-\alpha) q}} d x\right)^{1 / q} d y\right\}^{q} \\
& \leq \frac{1}{\lambda^{q}}\left\{\int_{|y|>2 a}|f(y)|\left(\int_{|x-y| \leq a} \frac{|\Omega(x)|^{q}}{|x|^{(n-\alpha) q}} d x\right)^{1 / q} d y\right\}^{q} \\
& \leq \frac{C}{\lambda^{q}}\left\{\int_{|y|>2 a}|f(y)| \frac{1}{|y|^{n-\alpha}}\left(\int_{|x-y| \leq a}|\Omega(x)|^{q} d x\right)^{1 / q} d y\right\}^{q} \\
& \leq \frac{C}{\lambda^{q}}\left\{\int_{|y|>2 a}|f(y)| \frac{1}{|y|^{n-\alpha}}\left(\int_{|y|-a}^{|y|+a} \int_{S^{n-1}}|\Omega(\theta)|^{q} d \theta r^{n-1} d r\right)^{1 / q} d y\right\}^{q} \\
& \leq \frac{C}{\lambda^{q}}\left\{\int_{|y|>2 a}|f(y)| \frac{1}{|y|^{n-\alpha}}\|\Omega\|_{q}\left(a|y|^{n-1}\right)^{1 / q} d y\right\}^{q} \\
& =C\|\Omega\|_{q}^{q}\left\{\frac{1}{\lambda} \int_{|y|>2 a}|f(y)|\left(\frac{a}{|y|}\right)^{1 / q} d y\right\}^{q},
\end{aligned}
$$

where $\|\Omega\|_{q}^{q}=\int_{S^{n-1}}|\Omega(\theta)|^{q} d \theta$. Thus, the conclusion of Theorem 1 immediately follows from Lemma 1.

It is easy to see that the conclusion of Theorem 2 is a direct consequence of Theorem 1 and Lemma 2.

\section{ACKNOWLEDGEMENT}

The author would like to thank the referee for his very valuable comments.

\section{REFERENCES}

1. S. Chanillo, D. Watson and R. L. Wheeden, Some integral and maximal operator related to starlike sets, Studia Math. 107 (1993), 223-255. MR 94j:42027

2. B. Muckenhoupt and R. L. Wheeden, Weighted norm inequalities for singular and fractional integrals, Trans. Amer. Math. Soc. 161 (1971), 249-258. MR 44:3155

3. F. Soria and G. Weiss, A remark on singular integrals and power weights, Indiana Univ. Math. Jour. 43 (1994), 187-204. MR 95g:42028

Department of Mathematics, Nanchang Vocational and Technical Teacher's College, Nanchang, Jiangxi, 330013, People's Republic of China

Current address: No. 35, Xianshi One Road, Nanchang, Jiangxi, 330006, People's Republic of China 